
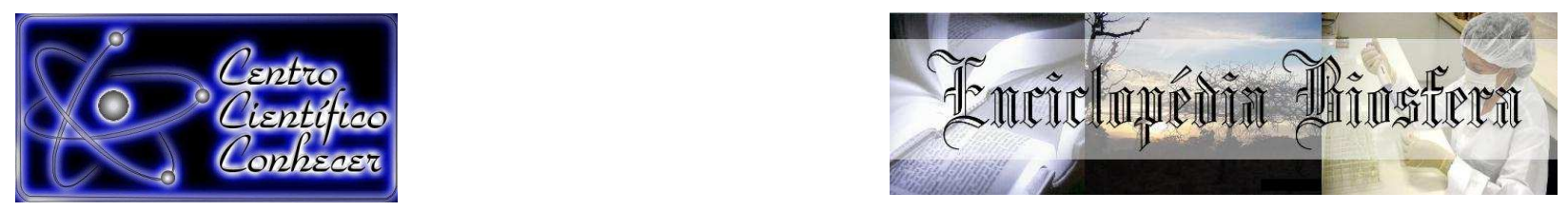

\title{
COMPORTAMENTO GERMINATIVO DE SEMENTES DE EUCALIPTO EM DUAS TEMPERATURAS COM O USO DE SAIS INORGÂNICOS
}

\author{
Abimael Gomes da Silva', Rita de Cássia Marino de Paula², \\ Flávio Ferreira da Silva Binotti ${ }^{3}$, Edilson Costa ${ }^{3}$
}

1 - Biólogo, mestrando na Universidade Estadual de Mato Grosso do Sul, UEMS,

Unidade Universitária de Cassilândia (maeldruida@hotmail.com);

2 - Engenheira Florestal, mestrando na Universidade Estadual de Mato Grosso do

Sul, UEMS, Unidade Universitária de Cassilândia,

3 - Professor Adjunto UEMS, Unidade Universitária de Cassilândia

Recebido em: 08/04/2017 - Aprovado em: 10/06/2017 - Publicado em: 20/06/2017

DOI: 10.18677/EnciBio_2017A33

\begin{abstract}
RESUMO
O nitrato de cálcio e o nitrato de potássio podem ser utilizados no tratamento de sementes para favorecer rotas metabólicas que contribua no processo germinativo. O objetivo desse estudo foi avaliar o efeito de sais inorgânicos (nitrato de potássio e cálcio), em dois experimentos, um em condições normais de temperatura e outro em temperatura sub-ótima durante a germinação, no potencial fisiológico de sementes de Eucalyptus grandis. Os experimentos utilizaram temperaturas controladas, sendo o primeiro a $25{ }^{\circ} \mathrm{C}$ e o segundo a $18{ }^{\circ} \mathrm{C}$ (temperatura sub-ótima). Ambos os experimentos foram realizados em delineamento inteiramente casualizado, constituídos de 4 tratamento e 5 repetições, as quais receberam $0,10 \mathrm{~g}$ de semente em cada repetição. Em ambos os experimentos, os tratamentos foram constituídos pelo umedecimento do substrato de germinação do tipo mata-borrão com as diferentes soluções: nitrato de cálcio a $0,2 \%$, nitrato de potássio a $0,2 \%$, a mistura de nitrato de cálcio a $0,2 \%$ + nitrato de potássio a $0,2 \%$, além do controle que foi realizado com água deionizada. Para sementes de $E$. grandis não é recomendado o uso de solução de nitrato de potássio a $0,2 \%$ no substrato de germinação, todavia o uso dessa solução no teste de germinação possibilita maior percentual de germinação, porém com menor velocidade.
\end{abstract}

PALAVRAS-CHAVE: Eucalyptusgrandis, nitrato de cálcio, nitrato de potássio.

\section{GERMINATING BEHAVIOR OF EUCALYPTUS SEEDS IN TWO TEMPERATURES WITH THE USE OF INORGANIC SALTS}

\begin{abstract}
Calcium nitrate and potassium nitrate can be used in the treatment of seeds to favor metabolic routes that contribute to the germination process. The objective of this study was to evaluate the effect of inorganic salts (potassium nitrate and calcium) in two experiments, one at normal temperature and the other at sub-optimal temperature during germination, on the physiological potential of Eucalyptus grandis seeds. The experiments used controlled temperatures, the first at $25^{\circ} \mathrm{C}$ and the second at $18^{\circ} \mathrm{C}$ (sub-optimal temperature). Both experiments were performed in a
\end{abstract}


completely randomized design, consisting of 4 treatments and 5 replicates, which received $0.10 \mathrm{~g}$ of seed in each replicate. In both experiments, the treatments were constituted by the wetting of the germination substrate of the blotter type with the different solutions: calcium nitrate $0.2 \%$, potassium nitrate $0.2 \%$, calcium nitrate mixture $0.2 \%$ + Potassium nitrate $0.2 \%$, in addition to the control that was performed with deionized water. For $\mathrm{E}$. grandis seeds the use of $0.2 \%$ potassium nitrate solution in the germination substrate is not recommended, however the use of this solution in the germination test allows a higher percentage of germination, but with a lower speed.

KEYWORDS: Eucalyptus grandis, calcium nitrate, potassium nitrate.

\section{INTRODUÇÃO}

O eucalipto é nativo da Austrália, Indonésia e Timor. Seu cultivo tem aumentado nos últimos anos e possui grande importância no segmento florestal, além de ser uma alternativa economicamente viável (COELHO et al., 2016; VIRGENS et al., 2016). A região do Mato Grosso do Sul apresentou, nos últimos cinco anos, cerca de 450 mil hectares plantados, sendo destaque entre os estados Brasileiros com maior área destinada ao eucalipto. O reflorestamento é uma prática que contribui para preservação de madeiras nativas e o seqüestro de carbono (IBÁ, 2016). O uso de madeiras de reflorestamento certificado é crescente na maioria dos países (ABRAF, 2013).

O crescimento do reflorestamento aumenta a demanda por mudas vigorosas. O uso de propagação vegetativa se popularizou pelo fato de anteriormente não haver controle de qualidade de sementes e os resultados eram geralmente plantios com mudas de baixa produtividade e stand desuniforme. O uso de sementes pode ser uma alternativa com menor custo quando comparado à propagação vegetal com maiores exigências técnicas (OLIVEIRA et al., 2013), resultado em mudas de qualidade proporcional à qualidade das sementes.

Diversas técnicas de tratamento de sementes visam assegurar a expressão do vigor resultando em mudas adequadas. Em sementes florestais essas técnicas podem contribuir para superação de dormência (KERBAUY, 2013) incorporar produtos que auxiliam nos processos germinativos ou na proteção da semente (MARCOS FILHO, 2015; SANTOS, 2016).

O nitrato de cálcio e o nitrato de potássio são conhecidos por influenciar muitas vezes o processo germinativo, podendo atuar também na superação de mecanismos de dormência em algumas sementes ou favorecer rotas metabólicas (KERBAUY, 2013; MARCOS FILHO, 2015). Os nitratos são substancias que liberam óxido nítrico, que atua como mensageiro de diversos processos metabólicos vegetais como o crescimento e desenvolvimento, proteção contra processos abióticos, reparação dos sistemas de membranas, etc. (SANZ et al., 2015). BINOTTI et al., (2014) observaram que o uso de nitrato de potássio promoveu o aumento de velocidade de germinação, além disso segundo BATISTA, (2016) as sementes de braquiária adquiriram maior tolerância a condições adversas com uso de nitrato de cálcio.

Uma das maneiras de avaliar a germinação é através do teste de germinação sendo que a primeira contagem do teste pode ser utilizada para avaliar o vigor das sementes, evidenciando que aquelas que apresentarem maior valor nesta avaliação, podem ser consideradas mais vigorosas (MARCOS FILHO, 2015). A temperatura 
pode interferir no processo de germinação alterando a velocidade de absorção de água e as reações bioquímicas desse processo (CARVALHO; NAKAGAWA, 2012).

Estudos de substâncias que liberam óxido nítrico em sementes florestais são pouco frequentes quando comparados aos feitos com sementes de hortaliças e braquiárias. Diante disso, a necessidade pelo conhecimento do comportamento germinativo em diferentes condições de temperatura com uso dessas substâncias na qualidade fisiológica de sementes é necessária. Esse ensaio teve por intuito gerar informações sobre o efeito de sais inorgânicos (nitrato de potássio e cálcio), em dois experimentos, um em condições normais de temperatura e outro em temperatura sub-ótima durante a germinação, no potencial fisiológico de sementes de Eucalyptus grandis.

\section{MATERIAL E MÉTODOS}

O experimento foi implantado na Universidade Estadual de Mato Grosso do Sul, Unidade Universitária de Cassilândia, no Laboratório de Análise de Sementes, em novembro de 2016. As sementes de Eucalyptus grandis foram adquiridas do Instituto Federal de Espécies Florestais.

Foram realizados dois experimentos em delineamento inteiramente casualizados, compostos de quatro tratamentos de cinco repetições com $0,10 \mathrm{~g}$ de sementes de E. grandis cada, como proposto em BRASIL (2009). Os experimentos foram realizados em câmara de germinação com temperaturas constantes, sendo uma a $18^{\circ} \mathrm{C}$, considerada como germinação em baixa temperatura (condição subótima) e o outra a $25^{\circ} \mathrm{C}$. Em ambos os experimentos os tratamentos foram constituídos pelo umedecimento do substrato de germinação do tipo mata-borrão com as diferentes soluções: $\mathrm{Ca}\left(\mathrm{NO}_{3}\right)_{2} \mathrm{0}, 2 \%, \mathrm{KNO}_{3} 0,2 \%$, a mistura de $\mathrm{Ca}\left(\mathrm{NO}_{3}\right)_{2}$ $0,2 \%+\mathrm{KNO}_{3} 0,2 \%$ e o controle que foi realizado com água deionizada.

O substrato utilizado para a germinação foram dois papéis do tipo mataborrão umedecido com quantidade de água equivalente ao triplo de sua massa, em caixas plásticas do tipo gerbox. Ao quinto dia foi realizada a primeira contagem (PC) e ao décimo quarto a última contagem (BRASIL, 2009) procedendo com a avaliação de germinação total $(G)$, além do cálculo do Índice de Velocidade de Germinação (IVG) por adaptação da fórmula de MAGUIRE (1962). Todos os dados submetidos ao teste da diferença mínima significativa, de LSD a $5 \%$ de probabilidade.

\section{RESULTADOS}

Foi observado que os resultados da primeira contagem, germinação total e índice de velocidade de germinação foram influenciados pelo fornecimento de sais inorgânicos no substrato de germinação (Tabela 1). O fornecimento de sais inorgânicos (nitrato de potássio e cálcio) no substrato de germinação propiciou menor percentual aos cinco dias (primeira contagem de germinação). Os sais alteraram o potencial hídrico do substrato afetando de forma negativa 0 estabelecimento de plântulas na primeira contagem.

$\mathrm{Na}$ avaliação de germinação total (14ํำ dias), verificaram-se valores menores de percentual de germinação para o tratamento com nitrato de potássio, os maiores valores foram obtidos com o uso de nitrato de cálcio. Foi observado por BATISTA et al., . (2015) que os tratamentos com nitrato de potássio ou nitrato de cálcio em sementes de pimenteira produziram mudas vigorosas quando comparadas com aquelas que não originaram de sementes tratadas. O uso de substâncias que liberam óxido nítrico, tais como o nitrato de potássio e o nitroprussiato de sódio 
promoveram benefício no processo germinativo de sementes sob estresse abiótico e permitem elevada expressão de vigor (ATAÍDE et al., 2015; KAISER et al., 2016).

A velocidade de germinação variou de 6 a 12 e foram menores nos tratamentos com fornecimento de sais inorgânicos no substrato de germinação. A presença do sal afeta o potencial hídrico do substrato, deixando o mesmo mais negativo, que propicia uma absorção de água pelas sementes mais lenta, consequentemente afeta a velocidade de germinação.

TABELA 1 - Primeira contagem de germinação (PC), germinação total (G) e índice de velocidade de germinação (IVG) a $25^{\circ} \mathrm{C}$ em função do uso de sais inorgânico no substrato de germinação. Cassilândia-MS, 2016.

\begin{tabular}{lccc}
\hline \multicolumn{1}{c}{ Temperatura do teste a $\mathbf{2 5} \mathbf{5}^{\circ} \mathbf{C}$} \\
\hline TRATAMENTO & $\mathbf{P C}(\%)$ & $\mathbf{G}(\%)$ & IVG \\
\hline Controle & $30 \mathrm{a}$ & $99 \mathrm{bc}$ & $12 \mathrm{a}$ \\
$\mathrm{KNO}_{3}$ & $3 \mathrm{~b}$ & $78 \mathrm{C}$ & $6 \mathrm{~b}$ \\
$\mathrm{Ca}\left(\mathrm{NO}_{3}\right)_{2}$ & $6 \mathrm{~b}$ & $111 \mathrm{a}$ & $9 \mathrm{~b}$ \\
$\mathrm{KNO}_{3}+\mathrm{Ca}\left(\mathrm{NO}_{3}\right)_{2}$ & $4 \mathrm{~b}$ & $89 \mathrm{bc}$ & $7 \mathrm{~b}$ \\
\hline $\mathrm{DMS}$ & 5,77 & 17,12 & 1,59 \\
\hline $\mathrm{CV} \%$ & 44,64 & 15,02 & 16,25 \\
\hline
\end{tabular}

Médias seguidas de mesma letra na coluna, não diferem entre si pelo teste LSD a $5 \%$ de probabilidade.

De acordo com a Tabela 2, a avaliação de primeira contagem de germinação, aos cinco dias, não apresentou plântulas originadas da germinação das sementes. As reações bioquímicas do processo germinativo são influenciadas pela temperatura. Essas reações controlam o crescimento da radícula, além de todo desenvolvimento do embrião, influenciando também a velocidade e porcentagem da germinação (CARVALHO \& NAKAGAWA, 2012). A germinação total apresentou o menor valor para o tratamento com nitrato de potássio, os demais tratamentos foram semelhantes estatisticamente ao tratamento controle.

O índice de velocidade de germinação (IVG) variou de 4 a 6 (Tabela 1), a utilização do nitrato de potássio no substrato de germinação proporcionou a menor velocidade de germinação, os demais tratamentos não interferiram nessa variável, visto que seus valores não diferem do tratamento controle.

Com os dois ensaios se verificou que a temperatura é fator limitante para o processo da germinação, sendo que aos $18{ }^{\circ} \mathrm{C}$ não foi possível observar sementes germinadas na primeira contagem enquanto que aos $25^{\circ} \mathrm{C}$ observou-se cerca de 30 sementes germinadas no controle. O uso de nitrato de cálcio não beneficiou o processo germinativo em condições sub-ótima $\left(18^{\circ} \mathrm{C}\right)$ de temperatura, como ocorreu em condições recomendas de germinação (25 ํ) $)$.

Segundo PECHE et al., (2016) o desenvolvimento vegetal responde de maneira distinta para as faixas de temperaturas. SILVA et al. (2016) observaram em ensaio com diferentes temperaturas e potenciais hídricos no desenvolvimento de Chorisiaglaziovii que as temperaturas abaixo de $20^{\circ} \mathrm{C}$ resultaram em plântulas com raízes de menores fitomassas secas. 
TABELA 2 - Primeira contagem germinação (PC), germinação total $(G)$ e índice de velocidade de germinação (IVG) a $18{ }^{\circ} \mathrm{C}$ em função do uso de sais inorgânico no substrato de germinação. Cassilândia-MS, 2016.

\begin{tabular}{lccc}
\hline & Temperatura do teste a $\mathbf{1 8}$ & \\
\hline TRATAMENTO & PC (\%) & $\mathbf{G}(\%)$ & IVG \\
\hline Controle & 0 & $90 \mathrm{a}$ & $6 \mathrm{a}$ \\
$\mathrm{KNO}_{3}$ & 0 & $56 \mathrm{~b}$ & $4 \mathrm{~b}$ \\
$\mathrm{Ca}\left(\mathrm{NO}_{3}\right)_{2}$ & 0 & $78 \mathrm{a}$ & $6 \mathrm{a}$ \\
$\mathrm{KNO}_{3}+\mathrm{Ca}\left(\mathrm{NO}_{3}\right)_{2}$ & 0 & $84 \mathrm{a}$ & $6 \mathrm{a}$ \\
\hline $\mathrm{DMS}$ & 0 & 19,5 & 1,51 \\
\hline $\mathrm{CV} \%$ & 0 & 19,37 & 20,85 \\
\hline
\end{tabular}

Médias seguidas de mesma letra na coluna, não diferem entre si pelo teste LSD a 5\% de probabilidade.

Ambos os experimentos evidenciaram que para as sementes de E. grandis o uso do nitrato de potássio $0,2 \%$ foi prejudicial no percentual e velocidade de germinação, não sendo recomendado. Apesar de efeitos conhecidos de algumas substâncias, o uso em algumas espécies vegetais não provocam resultados próximos aos esperados (PECHE et al., 2016), sendo preciso mais estudos para elucidar os mecanismos envolvidos.

\section{CONCLUSÃO}

Pode-se concluir que para sementes de $E$. grandis não é recomendado o uso de solução de nitrato de potássio a $0,2 \%$ no substrato de germinação, todavia o uso dessa solução no teste de germinação possibilita maior percentual de germinação, porém com menor velocidade.

\section{AGRADECIMENTOS}

Os autores agradecem à Fundação de Apoio ao Desenvolvimento do Ensino, Ciência e Tecnologia do Estado do Mato Grosso do Sul (Fundect) pela bolsa concedida ao primeiro autor e à CAPES pela bolsa concedida ao segundo autor e pelo apoio financeiro (FUNDECT/CNPq/PRONEM - MS, Processo 59/300.116/2015 - № FUNDECT 080/2015).

\section{REFERENCIAS}

Anuário estatístico da Assossiação de Produtores de Florestas Plantadas do Brasil (ABRAF). Disponível em: <http://www.ipef.br/estatisticas/relatorios/anuarioABRAF13-BR.pdf>.

ATAÍDE, G. M.; BORGES, E. E. L.; FLORES, A. V.; CASTRO, R. V. O. Óxido nítrico na germinação de sementes de baixo vigor de Dalbergianigra. Revista de Ciências Agrárias, Recife, v. 38, n. 3, p. 438-444, 2015. Disponível em: <http://www.scielo.mec.pt/pdf/rca/v38n3/v38n3a19.pdf>.

BATISTA, T. B.; BINOTTI, F. F. S.; CARDOSO, E.D.; BARDIVIESSO, E. M.; COSTA, E.; Aspectos fisiológicos e qualidade de mudas da pimenteira em resposta ao vigor e condicionamento das sementes. Bragantia, Campinas, v. 74, n. 4, p. 367-373, 2015. Disponível em: <http://dx.doi.org/10.1590/1678-4499.0133>. doi: 10.1590/16784499.0133 
BATISTA, T. B.; CARDOSO, E.D.; BINOTTI, F. F. S.; COSTA, E.; SÁ, M. E. Priming and stress under high humidity and temperature on the physiological quality of Brachiaria brizantha cv. MG-5 seeds. Acta Scientiarum, Maringá, v. 38, n. 1, p. $123-$ 127, 2016. Disponível em: <http://dx.doi.org/10.4025/actasciagron.v38i1.26021>. doi: 10.4025/actasciagron.v38i1.26021

BINOTTI, F. F. S.; SUEDA JUNIOR, C. I.; CARDOSO, E. D.; HAGA, K. I. NOGUEIRA D. C. Tratamentos pré-germinativos em sementes de Brachiaria. Revista Brasileira de Ciências Agrárias, Recife, v.9, n.4, p.614-618, 2014. Disponível em: <http://dx.doi.org/10.5039/agraria.v9i4a2781>. doi: 10.5039/agraria.v9i4a2781

BRASIL. Ministério da Agricultura e da Reforma Agrária. Regras para Análise de Sementes. Brasília: Mapa/ACS, 2009. 399p.

CARVALHO, N. M.; NAKAGAWA, J. Sementes: tecnologia e produção. 5. ed. Jaboticabal: Funep, 2012, 590p.

COELHO, R. M.; LEITE, A. M. P.; LEONEL, M. S.; MATUDA, J. J.; FREITAS, L. C. Avaliação econômica do uso da madeira de eucalipto para diferentes finalidades, na região do Alto Jequitinhonha, MG. Floresta, Curitiba, v. 46, n. 2, p. 155 - 164, 2016. Disponível em: <http://dx.doi.org/10.5380/rf.v46i2.38600>. doi: 10.5380/rf.v46i2.38600

Indústria Brasileira de Árvores - IBÁ. Relatório IBÁ 2016. Brasília; 2015. 100p. Disponível em: <http://iba.org/images/shared/Biblioteca/IBA_RelatorioAnual2016_.pdf>.

KAISER, I. S.; MACHADO, L. C.; LOPES, J. C.; MENGARDA, L. H. Efeito de liberadores de óxido nítrico na qualidade fisiológica de sementes de repolho sob salinidade. Revista Ceres, Viçosa, v. 63, n.1, p. 039-045, 2016. Disponível em: <http://dx.doi.org/10.1590/0034-737X201663010006>. doi: 10.1590/0034737X201663010006

KERBAUY, G. B. Fisiologia Vegetal. Rio de Janeiro, RJ, 2 Ed. Guanabara Koogan, 2013. $431 p$.

LOPES, H. M.; MENEZES, B. R. S.; SILVA, E. R. S.; RODRIGUES, D. L. Condicionamento fisiológico de sementes de cenoura e pimentão. Revista Brasileira de Agrociência, Pelotas, v. 17, n. 3-4, p. 296-302, 2011. Disponível em: <https://periodicos.ufpel.edu.br/ojs2/index.php/CAST/article/view/2062/1900>. doi: HTTP://DX.DOI.ORG/10.18539/CAST.V17I3.2062

MARCOS FILHO, J. Fisiologia de sementes de plantas cultivadas. Piracicaba, SP: FEALQ, 2015. 655 p.

MAGUIRE, J.D. Speed of germination and in selection and evaluation for seedling emergence and vigor. Crop Science, v.2, n.2, p.176-177, 1962. Disponível em: <https://dl.sciencesocieties.org/publications/cs/abstracts/2/2/CS0020020176>.

OLIVEIRA, L. S.; DIAS, P. C.; BRONDANI, G. E. Micropropagação de espécies florestais brasileiras. Pesquisa Florestal Brasileira, Colombo, v. 33, n. 76, p. 439- 
453, 2013. Disponível em: <http://dx.doi.org/10.4336/2013.pfb.33.76.481>. doi: 10.4336/2013.pfb.33.76.481

PECHE, P. M.; BARBOSA, C. M.A; PIO, R.; SOUZA, P. H. A.; VALLE, M. H. Estratificação das sementes, ácido giberélico e temperatura na obtenção de portaenxertos de caquizeiros. Revista Ciência Agronômica, Fortaleza, v. 47, n. 2, p. 387-392, 2016. Disponível em: <https://doi.org/10.5935/1806-6690.20160046>. doi: 10.5935/1806-6690.20160046

PEREIRA, A. G.; CRUZ, E. D.; BARROS, H. S. D. Métodos para superação da dormência em sementes de Stryphnodendron pulcherrimum. Pesquisa florestal brasileira, Colombo, v. 36 , n. 87 , p. 195-199, 2016. Disponível em: <http://dx.doi.org/10.4336/2016.pfb.36.87.931>. doi: 10.4336/2016.pfb.36.87.931

SANTOS, S. R. G. Peletização de Sementes Florestais no Brasil: Uma Atualização. Floresta e Ambiente, Seropédica, v. 23 n. 2, p. 286-294, 2016. Disponível em: $<$ https://doi.org/10.1590/2179-8087.120414>. doi: 10.1590/2179-8087.120414

SANZ, L.; ALBERTOS, P.; MATEOS, I.; SÁNCHEZ-VICENTE, I.; LECHÓN, T.; FERNÁNDEZ-MARCOS, M.; LORENZO, O. Nitric oxide (NO) and phytormones crosstalk during early plant development. Journal of Experimental Botany, v. 66, n. 10, p. 2857-2868, 2015. Disponível em: <https://doi.org/10.1093/jxb/erv213>. doi: $10.1093 / j x b / e r v 213$

SILVA, M. L. M.; ALVES, E. U.; BRUNO, R. L. A.; SANTOS-MOURA, S. S.; SANTOS NETO, A. P. Germinação de sementes de Chorisiaglaziovii O. Kuntze submetidas ao estresse hídrico em diferentes temperaturas. Ciência Florestal, Santa Maria, v. 26, n. 3, p. 999-1007, 2016. Disponível em: <http://dx.doi.org/10.5902/1980509824229>. doi: $10.5902 / 1980509824229$

VIRGENS, A. P.; FREITAS, L. C.; LEITE, A. M. P. Análise Econômica e de Sensibilidade em um Povoamento Implantado no Sudoeste da Bahia. Floresta e Ambiente, Seropédica, v.23, n. 2, p. 211-219, 2016. Disponível em: <http://floram.org/files/v23n2/floramAO104914.pdf>. doi: 10.1590/2179-8087.104914 